\title{
Genetic Relationships in the Toxin-Producing Fungal Endophyte, Alternaria oxytropis Using Polyketide Synthase and Non-Ribosomal Peptide Synthase Genes
}

\author{
Rebecca Creamer ${ }^{1, *}$, Deana Baucom Hille ${ }^{1}$, Marwa Neyaz ${ }^{1}\left(\mathbb{D}\right.$, Tesneem Nusayr ${ }^{2}$, Christopher L. Schardl $^{3} \mathbb{D}^{\mathbb{D}}$ \\ and Daniel Cook ${ }^{4}$ \\ 1 Department of Entomology, Plant Pathology and Weed Science, New Mexico State University, \\ Las Cruces, NM 88003, USA; dlhille2017@gmail.com (D.B.H.); marwane@nmsu.edu (M.N.) \\ 2 Arts and Science Biology Department, University of Houston, Victoria, TX 77901, USA; tnusayr@gmail.com \\ 3 Department of Plant Pathology, University of Kentucky, Lexington, KY 40508, USA; schardl@uky.edu \\ 4 Poisonous Plant Laboratory, US Department of Agriculture, Logan, UT 84341, USA; \\ Daniel.cook@ars.usda.gov \\ * Correspondence: creamer@nmsu.edu
}

Citation: Creamer, R.; Hille, D.B.; Neyaz, M.; Nusayr, T.; Schardl, C.L.; Cook, D. Genetic Relationships in the Toxin-Producing Fungal Endophyte, Alternaria oxytropis Using Polyketide Synthase and Non-Ribosomal Peptide Synthase Genes. J. Fungi 2021, 7, 538. https://doi.org/10.3390/jof7070538

Academic Editor: Willem Melchers

Received: 7 June 2021

Accepted: 30 June 2021

Published: 6 July 2021

Publisher's Note: MDPI stays neutral with regard to jurisdictional claims in published maps and institutional affiliations.

Copyright: (c) 2021 by the authors. Licensee MDPI, Basel, Switzerland. This article is an open access article distributed under the terms and conditions of the Creative Commons Attribution (CC BY) license (https:// creativecommons.org/licenses/by/ $4.0 /)$.

\begin{abstract}
The legume Oxytropis sericea hosts a fungal endophyte, Alternaria oxytropis, which produces secondary metabolites (SM), including the toxin swainsonine. Polyketide synthase (PKS) and nonribosomal peptide synthase (NRPS) enzymes are associated with biosynthesis of fungal SM. To better understand the origins of the SM, an unannotated genome of $A$. oxytropis was assessed for protein sequences similar to known PKS and NRPS enzymes of fungi. Contigs exhibiting identity with known genes were analyzed at nucleotide and protein levels using available databases. Software were used to identify PKS and NRPS domains and predict identity and function. Confirmation of sequence for selected gene sequences was accomplished using PCR. Thirteen PKS, 5 NRPS, and 4 PKSNRPS hybrids were identified and characterized with functions including swainsonine and melanin biosynthesis. Phylogenetic relationships among closest amino acid matches with Alternaria spp. were identified for seven highly conserved PKS and NRPS, including melanin synthesis. Three PKS and NRPS were most closely related to other fungi within the Pleosporaceae family, while five PKS and PKS-NRPS were closely related to fungi in the Pleosporales order. However, seven PKS and PKSNRPS showed no identity with fungi in the Pleosporales or the class Dothideomycetes, suggesting a different evolutionary origin for those genes.
\end{abstract}

Keywords: Alternaria sect. Undifilum; polyketide synthase; secondary metabolite; nonribosomal peptide synthase; swainsonine biosynthesis

\section{Introduction}

The endophytic fungus Alternaria (section Undifilum) oxytropis (Q. Wang, Nagao and Kakish.) Woudenb and Crous produces many secondary metabolites, including swainsonine, a compound toxic to mammals. This and related Alternaria sect. Undifilum spp. (family Pleosporaceae, order Pleosporales, class Dothideomycetes) are found within locoweeds, Astragalus and Oxytropis species that contain swainsonine. When these plants are ingested by mammals such as cattle, horses, sheep, and goats, normal cellular function is disrupted resulting in locoism disease ([1,2]. Other fungi, including Slafractonia leguminicola (order Pleosporales), Metarhizium anisopliae (class Sordariomycetes), members of the Arthodermataceae, and a yet undescribed Chaetothyrialean endophyte from Ipomoea carnea also produce swainsonine [3-5]. The biosynthetic pathway for swainsonine has been partially characterized that includes a gene cluster SWN which include a hybrid nonribosomal peptide synthetase (NRPS)-reducing ketide synthase swnK that was demonstrated to be essential for swainsonine biosynthesis in Metarhizium robertsii using a knockout of the gene 
through homologous gene replacement that lacked swainsonine production and complementation that restored its presence [6]. This work identified a SWN gene cluster associated with swainsonine biosynthesis in Alternaria oxytropis, S. leguminicola, Arthroderma and the Chaeothyriales fungus associated with Ipomoea carnea, and showed that the order of the different domains within swnK was identical among all the swainsonine-producing fungi, including the Chaetothyriales endophyte from Ipomoea carnea, Metarhizium spp., and the dermatophytes. Noor et al. [7] demonstrated that the KS portion of PKS-NRPS is highly conserved among all swainsonine-producing Alternaria spp., but differed slightly between plant pathogens and nonpathogens.

Many fungi produce specialized secondary metabolites (SM) for virulence, defense, or communication [8]. Biosynthetic pathways of many SMs include steps catalyzed by multifunctional polyketide synthase (PKS) enzymes. These enzymes direct the structure and biosynthesis of the compounds produced and contain multiple domains leading to a high diversity of SMs [9]. The high diversity in SMs obscures understanding of how SM pathways have evolved in fungi. In addition to duplications, domain shuffling, neofunctionalization, and subfunctionalization, some SM gene clusters are suggested to be transferred between diverse fungi by horizontal (lateral) gene transfer (HGT) [8].

Genes associated with toxin biosynthetic pathways in fungi have been found to encode PKSs and NRPSs $[10,11]$. They are often clustered with genes encoding other steps in the same pathway and can be identified from genomes using prediction software based on PKS domains [12]. Different functional types of PKSs can be identified from sequence and domain structure. Fungi can have type I PKSs, of which there can be highly reducing (HR), non-reducing (NR), or partially reducing (PR) categories, all of which begin with simple carboxylic acids and are iterative [13]. In addition, fungi have NRPSs that use amino acids as starter units for building compounds. Hybrids involving both PKS and NRPS domains have also been identified in fungi and catalyze production of many different polyketide and amino acid hybrid compounds [14].

Genome mining of toxin-producing Fusarium spp. (class Sordariomycetes) for SM genes has revealed 30, 32, 28, and 26 PKS and NRPS gene clusters for F. graminearum, F. verticillioides, F. oxysporum, and F. solani, respectively [15]. Examining the genomes of Aspergillus spp. has also revealed a range of genes. Using multiple software programs along with manual annotations, total clusters (including NRPS-like enzymes) predicted for A. nidulans, A. fumigatus, A. niger, and A. oryzae are 71, 39, 81, and 75, respectively [16]. SM genes have also been identified through genome analysis for the endophytic fungus Pestalotiopsis fici [17]. In P. fici, 27 PKSs, 12 NRPSs, and five PKS-NRPS hybrids have been identified [18]. Perhaps due to their growth habits, endophytes may have smaller numbers of PKSs compared to Fusarium and Aspergillus.

To better understand the origins of PKS, NRPS, and PKS-NRPS genes, we investigated their presence in the swainsonine-producing fungus, Alternaria oxytropis. We sought to use genomic analyses to identify PKSs, NRPSs, and hybrid PKS-NRPSs, and predict their structures and functions. The evolutionary relationships among these genes suggest diverse origins for SMs.

\section{Materials and Methods}

The genome of A. oxytropis isolated from Oxytropis sericea collected from Raft River, UT in 1979 was sequenced using Illumina MiSeq with 250 bp paired-end reads on a 400 bp library in Advanced Genetics Technology Center at the University of Kentucky as reported in Cook et al. [6]. Assembly was done using CLC Genomics Workbench 8.0.2 (Qiagen, Germantown, MD, USA). An unannotated, partially assembled genome (due to high number of repeated sequence) was obtained. For this work, the partially assembled genome was made into a searchable database in Geneious v. R8 (Geneious, CA, USA) [19]. To identify contigs containing PKS genes, several known nucleotide sequences were used to search the database using the parameters of a discontiguous megablast. Thirty nucleotide sequences of fungal PKS genes were used as query for matching contigs in the A. oxytropis 
genome including three obtained from the biosystems database at the National Center for Biotechnology Information (NCBI) and 27 from Clustermine 360, the database of microbial PKS/NRPS [20,21].

Each contig that was identified from the initial search was analyzed for nucleotide sequence similarity using a discontiguous megablast search function of the nucleotide redundant database of NCBI. Contigs containing partial sequence of PKS genes were completed by searching the A. oxytropis genome for similar matches and manual assembly of contigs within the Geneious software. Maximum parsimony analyses of A. oxytropis PKS gene contigs along with Clustermine360 fungal PKS genes was used to identify duplicate sequences and determine PKS types (Paup, 1000 bootstrap). All contigs containing Pkslike genes were characterized through BLASTn, BLASTp, Smartblast, and Conserved Domain Database searches of the NCBI database along with analysis through antibiotics and Secondary Metabolite Analysis SHell (antiSmash) v. 3.0 and Secondary Metabolites Unknown Regions Finder (SMURF) [22].

Various programs were used to analyze NRPS and PKS genes and domains. AntiSmash was also used to predict NRPS, PKS and secondary metabolites [23,24]. Natural Product Domain Seeker (NaPDoS) was used to identify and predict KS and C domains [25], and Non-ribosomal Peptide Synthase Substrate Predictor (NRPSsp) was used to predict NRPS substrates [26]. InterProScan was used to form predictive models [27], and Secondary Metabolite Prediction and Identification (SeMPI) was used to predict and identify pipelines for PKS and NRPS [28].

Confirmation of sequence for selected sequences was accomplished using PCR and by comparison with a second partial genome sequence of $A$. oxytropis. Primers were designed based on the obtained sequences in Geneious, and PCR was performed as previously reported [29]. All sequences were deposited into Genbank and accession numbers are listed in Table 1. Closest matches by BLASTp were based on percentage identity for sequences with query coverage greater that $90 \%$.

Table 1. Polyketide synthases and non-ribosomal peptide synthases of Alternaria section Undifilum oxytropis with highest Blastp hits and \% identity match of amino acid sequences.

\begin{tabular}{|c|c|c|c|c|c|c|}
\hline Type & Contig & $\begin{array}{l}\text { Genbank } \\
\text { Accession }\end{array}$ & $\%$ Identity & BLASTp Hit & Accession & Protein Match Category \\
\hline PKS-NRPS & 58882 & KY365741 & 81 & Slafractonia leguminicola & AQV04236 & $\mathrm{M}$ \\
\hline \multirow[t]{3}{*}{ hybrid } & 5601 & MK058350 & 69 & Setosphaeria turcica & XP_008025924 & $\mathrm{M}$ \\
\hline & 21438 & MK058352 & 88 & Pyrenophora teres & XP_003303559 & $\mathrm{M}$ \\
\hline & 62407 & MK058351 & 64 & $\begin{array}{l}\text { Tolypocladium } \\
\text { ophioglossoides }\end{array}$ & KND87119 & $\mathrm{N}$ \\
\hline \multirow{7}{*}{$\begin{array}{l}\text { Type I, highly } \\
\text { reducing }\end{array}$} & 2122 & MK471354 & 90 & Alternaria solani & Q5KTM9 & A \\
\hline & 1562 & MK495800 & 85 & Bipolaris maydis & AAR90260 & $\mathrm{P}$ \\
\hline & 59499 & MK492487 & 66 & Aspergillus nomius & KNG90368 & $\mathrm{N}$ \\
\hline & 96133 & MK492486 & 88 & Alternaria alternata & AFN68297 & A \\
\hline & 9132 & MK450599 & 73 & Fusraium aywerte & ALQ32761 & $\mathrm{N}$ \\
\hline & 39849 & MK492485 & 76 & Pyrenophora tritici-repentis & PZC93669 & M \\
\hline & 12778 & MK471353 & 65 & $\begin{array}{c}\text { Fusarium oxysporum } f . s p . \\
\text { conglutinans }\end{array}$ & EXL67078 & $\mathrm{N}$ \\
\hline Type I, partially & 17612 & MK495801 & 63 & $\begin{array}{l}\text { Cladosporium } \\
\text { cladosporioides }\end{array}$ & AOAOYOM151 & $\mathrm{N}$ \\
\hline \multirow[t]{3}{*}{ reducing } & 8407 & MK492484 & 81 & Alternaria solani & Q2ABP6 & $\mathrm{A}$ \\
\hline & 3398 & MK492483 & 64 & Metarhizium brunneum & KID62944 & $\mathrm{N}$ \\
\hline & 42460 & MK507973 & 46 & Colletotrichum fructicola & ELA32259 & $\mathrm{N}$ \\
\hline Type I, & 40283 & MK402071 & 95 & Alternaria alternata & AFN68292 & A \\
\hline non-reducing & 29103 & MK471352 & 70 & Epicoccum nigrum & OSS52043 & M \\
\hline \multirow[t]{5}{*}{ NRPS } & 33635 & MK409655 & 91 & Alternaria alternata & AFN69082 & A \\
\hline & 5682 & MK468800 & 91 & Alternaria alternata & XP_0188382376 & A \\
\hline & 7859 & MK426697 & 76 & Bipolaris maydis & AAX09991 & $\mathrm{P}$ \\
\hline & 8194 & MK468799 & 87 & Pyrenophora tritici-repentis & XP_001939433 & $\mathrm{P}$ \\
\hline & 40703 & MK471351 & 82 & Alternaria alternata & XP_018389223 & A \\
\hline
\end{tabular}

\% query cover (percentage of the protein that was included in the match) averaged 99.1\% (range 97-100); A = Alternaria genus 1-4 >Pleosporacae family; $\mathrm{P}=$ Pleosporaceae family 1-4; $\mathrm{M}=$ Pleosporales order 1-2 > not Dothidiomycetes class; $\mathrm{N}=$ not Pleosporales order/not Dothidiomycetes class. 
The highest seven matches for each amino acid sequence using BLASTp were used for generating phylogenetic trees. Sequences were aligned with MUSCLE using Geneious 10.0.9 software and trees were generated using PAUP and maximum parsimony with 100 replicates. Outgroups are marked on each tree and were the sixth closest matches for each tree.

\section{Results}

\subsection{Phylogenetic Framework}

Twenty-two PKS and NRPS genes, were identified from the A. oxytropis genome; 13 were identified as type I PKSs, five as NRPSs, and four as hybrid PKS-NRPSs. Each gene has been named as the functional type of gene followed by the original contig number from the genome search (Tables 1 and 2). Closest matches by BLASTp were based ranged from a low of $46 \%$ to a high of $95 \%$ identity for sequences with $97-100 \%$ query coverage. All but two of the samples had a query coverage of 99 or $100 \%$.

Table 2. SeMPI, antiSMASH, NaPDoS, NRPSep, and IntrProScan predictions for Alternaria oxytropis PKS and NRPS domains. The predictions show the presence or absence of condensation domain (CD), and ketosynthase domain (KSD); the predicted substrates for the adenylation domain; and the enzymatic cyclization of the final product.

\begin{tabular}{cccccc}
\hline Contig & $\begin{array}{c}\text { Genbank } \\
\text { Accession }\end{array}$ & CD & KSD & $\begin{array}{c}\text { Adenylation Domain } \\
\text { Substrate }\end{array}$ & Cyclization \\
\hline 58882 & KY365741 & - & + & Gly & No \\
5601 & MK058350 & + & + & Phe & No \\
21438 & MK058352 & + & + & Phe & No \\
62407 & MK058351 & + & + & Pyr & No \\
12778 & MK471353 & + & + & Phe & Cyclize \\
33635 & MK409655 & + & - & Pro, Pro, Phe & Likely \\
5682 & MK468800 & + & - & Pro, Try, Try & No \\
7859 & MK426697 & + & - & Try, Pro, Phe & Likely \\
8194 & MK468799 & + & - & Aminoadipate, Phe, Phe, Val & No \\
40703 & MK471351 & + & - & & \\
\hline
\end{tabular}

Several different domains were identified among the PKSs using software to predict domains including: ketoacyl synthase (PKS), acyl transferase (AT), dehydratase (DH), methyl transferase (MT), acyl carrier protein (ACP), enoyl reductase (ER), and ketoreductase (KR). Among the NRPSs the following were identified: adenylation (A), terminal reductase (TD), condensation (C), peptidyl carrier protein (PCP) (Supplemental Figure S1). Importantly, not all domains were identified in each gene of a particular PKS or NRPS.

\subsection{PKS-NRPS Hybrids}

Four hybrid PKS-NRPSs were identified, all were similar to a genus in the Ascomycota, two associated with plants and the other two associated with insects or animals, and only one has an established function (swainsonine biosynthesis) (Table 1).

The presence of swnK was verified for PKS-NRPS 58882 (Figure S1). Secondary metabolite product prediction also verified that PKS-NRPS 58882 was likely to produce swainsonine and establishing that the software was effective (Table 2). A BLASTn search verified that this PKS-NRPS was most similar to the swainsonine-producing fungi Slafractonia leguminicola, a Chaetothyriaceae, and several Metarhizium sp. Alignment identity for nucleic acid was 74\%, with the swnK gene of S. leguminicola, 70\% with the swainsonine biosynthesis gene cluster of Chaetothyriaceae, and 69\% with the same cluster in M. robertsii. A BLASTp search revealed matches to a polyketide synthase from Pyrenophora seminiperda (Pleosporaceae, Pleosporales, Dothideomycetes) (81\% amino acid identity), Slafractonia leguminicola (Pleosporales) SwnK (73\%), a polyketide synthase from Clohesyomyces aquaticus (Pleosporales) (71\%), and a polyketide synthase of Metarhizium acridum (Sordariomycetes) (69\%). PKS-NPRS 58882 had amino acid homology of $67-81 \%$ with a variety of fungi, and only the top three hits are within the order Pleosporales (Table 3, Figure 1). 
Table 3. Percent amino acid (aa) identity of pblast results of PKS-NRPS 58882 sequence. Fungal order within the class Dothideomycetes (D) or other fungal class as listed.

\begin{tabular}{cccc}
\hline Organism & Accession & Order/Class & \% aa Identity \\
\hline Pyrenophora seminiperda & RMZ73569.1 & Pleosporales/D & $80.9 \%$ \\
Slafractonia leguminicola & AQV04236.1 & Pleosporales/D & $72.7 \%$ \\
Clohesyomyces aquaticus & ORY11783.1 & Pleosporales/D & $71.0 \%$ \\
Metarhizium acridum & XP_007815889.1 & Sordariomycetes & $69.0 \%$ \\
Chaetothyriaceae & AQV04224.1 & Eurotiomycetes & $69.1 \%$ \\
Pseudogymnoascus sp. & KFY51099.1 & unknown/D & $67.3 \%$ \\
Rosellinia necatrix & GAP93000.1 & Sordariomycetes & $67.0 \%$ \\
\hline
\end{tabular}

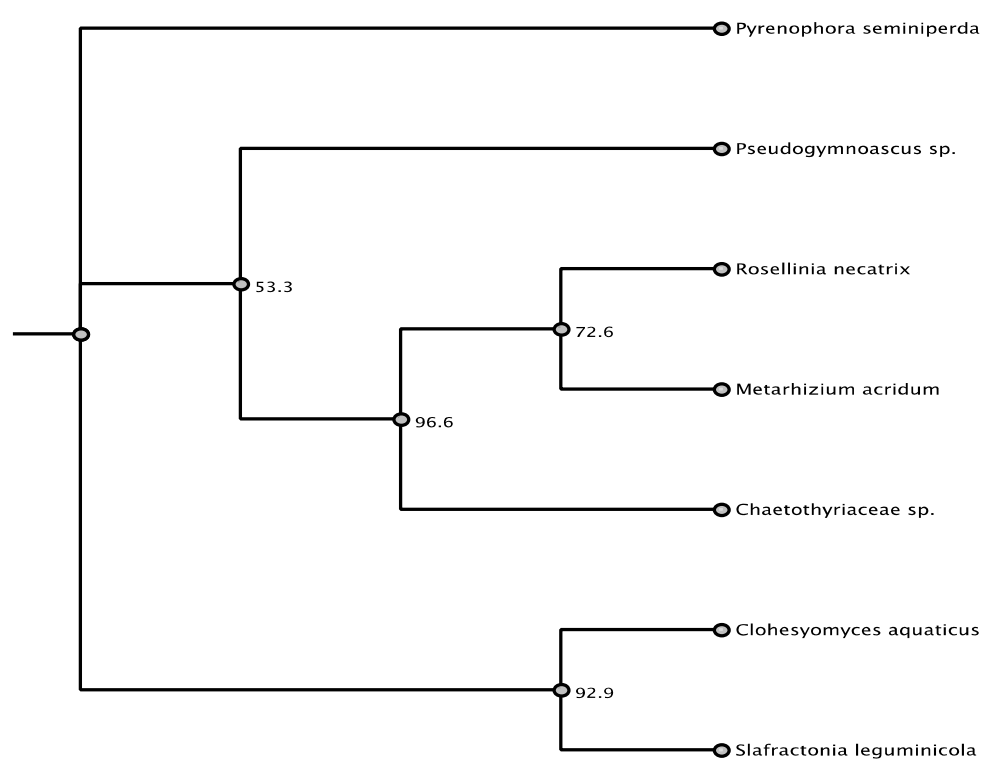

Figure 1. Maximum parsimony tree (MUSCLE alignment and 1000 replicates). Pseudogymnoascus sp. was used as the outgroup. Fungi in tree are pblast results of 58882 sequence.

PKS-NRPS 5601 was most similar to a hypothetical polyketide synthase of Setosphaeria turcica (Pleosporaceae, Pleosporales, Dothideomycetes) according to BLASTn and BLASTp (Figure S2). Alignments with the hypothetical mRNA and protein sequences resulted in identity matches of $67.1 \%$ and $69 \%$, respectively. The second closest BLASTp match did not fall within the Pleosporales and the remainder did not fall within the Dothideomycetes. PKS-NRPS 21438 was most similar to a hypothetical protein from Pyrenophora teres (Pleosporaceae, Pleosporales, Dothideomycetes) by BLASTn and BLASTp (Figure S3). Alignment identities were $87 \%$ for nucleic acid and $88 \%$ for amino acid. The closest match to PKS-NRP 21438 with a known function by BLASTp was Sch210972, that inhibits cytokine receptors, from Hapsidospora irregularis (Sordariomycetes) with $52 \%$ amino acid identity. Only the closest BLASTp match, Pyrenophora teres is within the Pleosporales (Table 4) and all the other matches fell within the Sordariomycetes at or below 52\% (Figure 2). PKS-NRPS 62407 was most similar by BLASTn to mRNA for hypothetical proteins from Uncinocarpus reesii (Eurotiomycetes) with $68.7 \%$ identity, and the top BLASTp hit was to a PKS-NRPS of Tolypocladium ophioglossoides (Sordariomycetes), with 64\% identity (Figure S4).

\subsection{Type I PKS}

Thirteen PKS-like genes were of the fungal iterative Type I PKS as characterized by linear organization with different levels of reducing capabilities depending on the presence or absence of beta-keto domains. Of the 13, seven were identified as highly reducing, four as partially reducing, and two as non-reducing (Table 1 ). Some of the type I PKSs of $A$. oxytropis are potential orthologs to PKSs of known function from other fungi. The majority of the others were closely related to genera from the Ascomycota that are associated with plants, but with unknown function identified for the particular PKSs. 
Table 4. Percent amino acid identity of pblast results of PKS-NRPS 21438 sequence. Fungal order if within the class Dothideomycetes (D) or other fungal class as listed.

\begin{tabular}{cccc}
\hline Organism & Accession & Order/Class & \% aa Identity \\
\hline Pyrenophora teres & EFQ87243.1 & Pleosporales/D & $87.5 \%$ \\
Acremonium chrysogenum & KFH40930.1 & Sordariomycetes & $52.7 \%$ \\
Hapsidospora irregularis & AKG54858.1 & Sordariomycetes & $52.2 \%$ \\
Pochonia chlyamydosporia & XP_018142849.1 & Sordariomycetes & $69.2 \%$ \\
Valsa mali var. pyri & KUI56379.1 & Sordariomycetes & $52.0 \%$ \\
Trichoderma guizhouense & OPB40675.1 & Sordariomycetes & $48.2 \%$ \\
Trichoderma harzianum & PKK52345.1 & Sordariomycetes & $48.0 \%$ \\
\hline
\end{tabular}

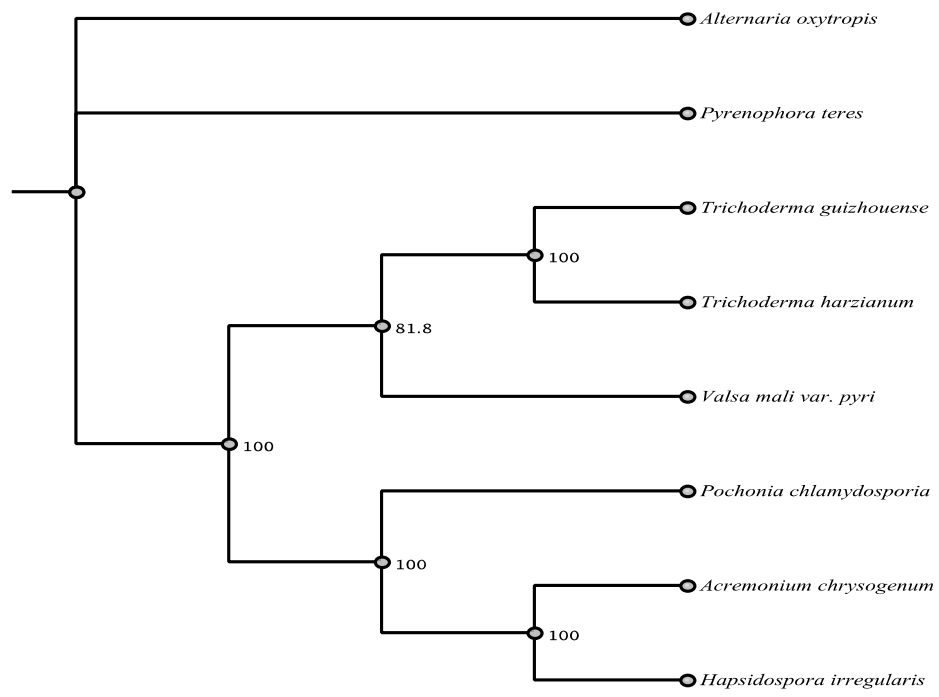

Figure 2. Maximum parsimony tree (MUSCLE alignment and 1000 replicates). Trichoderma guizhouense was used as the outgroup. Fungi in tree are pblast results of 21438 sequence.

\subsubsection{Highly Reducing Type I PKSs}

Among the identified highly reducing type I PKSs, PKS 2122 had the highest identity with a protein of known function. A megablast search of the NCBI nucleotide database identified highest identity $(83.8 \%)$ to the alt5 gene of Alternaria solani (Pleosporaceae, Pleosporales) and $90 \%$ amino acid identity. PKS 2122 was identified in both AntiSMASH and the CDD to have the domains KS, AT, DH, MT, ER, KR, and ACP as a type I, HR-PKS which are the same domains that are present in alt5 in the same order with a similar size of linker regions (Figure S5). The alt5 gene encodes for PKSN, the enzyme responsible for producing the compound alterapyrone [30]. PKS 2122 was very highly conserved and had very high amino acid identity of $89-90 \%$ with the top seven matches that were all Alternaria spp. or relatives (Table S1, Figure 3).

PKS 1562 was most similar to Bipolaris maydis (Pleosporaceae, Pleosporales) by BLASTn to $P k s 5$, a gene that encodes a PKS with no known function. PKS 1562 has the domains KS, AT, DH, MT and ER from the CDD. Alignment of PKS 1562 with PKS5 gave 85\% amino acid identity match. Comparing the amino acid sequences of both on the CDD showed that the KS, AT, and DH domains and the linking regions therein were highly similar whereas Pks5 contains a different MT domain as well as a KR domain (Figure S6). PKS 1562 has only a partial KR domain at the $3^{\prime}$ end.

Both highly reducing type I PKS 59499 and PKS96133 are similar to PKSs from other toxin producing species. PKS 59499 showed $67.7 \%$ identity in a BLASTn search with Aspergillus nomius (Eurotiomycetes) and an amino acid identity of 66\%. PKS 96133 was most closely related by BLASTn to $p k s F$ of Alternaria alternata (Pleosporaceae, Pleosporales) another known toxin producer (Figure S7). Alignment of DNA and proteins found identity of $80.1 \%$ and $88 \%$ between PKSF and PKS 96133. The domains KS, AT, DH, and MT were the same as seen with CDD comparison though the order was different (Figure S8). The 
function of PKSF is not known, but PKS 96133 was highly conserved and had very high amino acid identity of $85-88 \%$ with the top seven matches, which were all Alternaria spp. or other Pleoporales (Table 5, Figure 4).

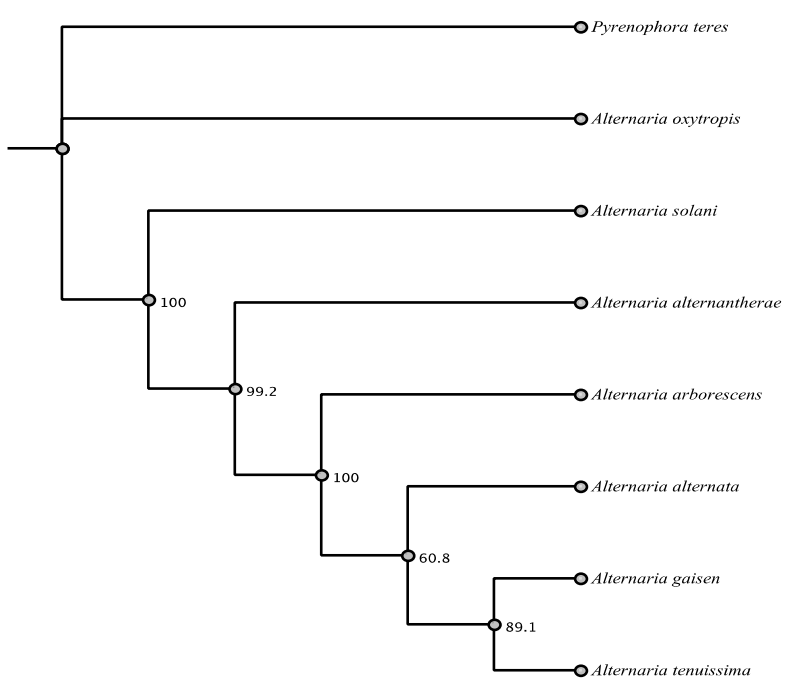

Figure 3. Maximum parsimony tree (MUSCLE alignment and 1000 replicates). Alternaria arborescens was used as the outgroup. Fungi in tree are pblast results of 2122 sequence.

Table 5. Percent amino acid (aa) identity of pblast results of PKS 96133 sequence. Fungal order within the class Dothideomycetes.

\begin{tabular}{cccc}
\hline Organism & Accession & Order & \% aa Identity \\
\hline Alternaria alternata & AFN68297.1 & Pleosporales & $87.7 \%$ \\
Stemphylium lycopersici & RAR01042.1 & Pleosporales & $85.4 \%$ \\
Alternaria tenuissima & RYN28010.1 & Pleosporales & $86.9 \%$ \\
Ascochyta rabiei & KZM21157.1 & Pleosporales & $86.6 \%$ \\
Phoma betae & BAQ25466.1 & Pleosporales & $86.1 \%$ \\
Bipolaris maydis & AAR90270.1 & Pleosporales & $85.9 \%$ \\
Exserohilum turcica & XP_008031663.1 & Pleosporales & $87.1 \%$ \\
\hline
\end{tabular}

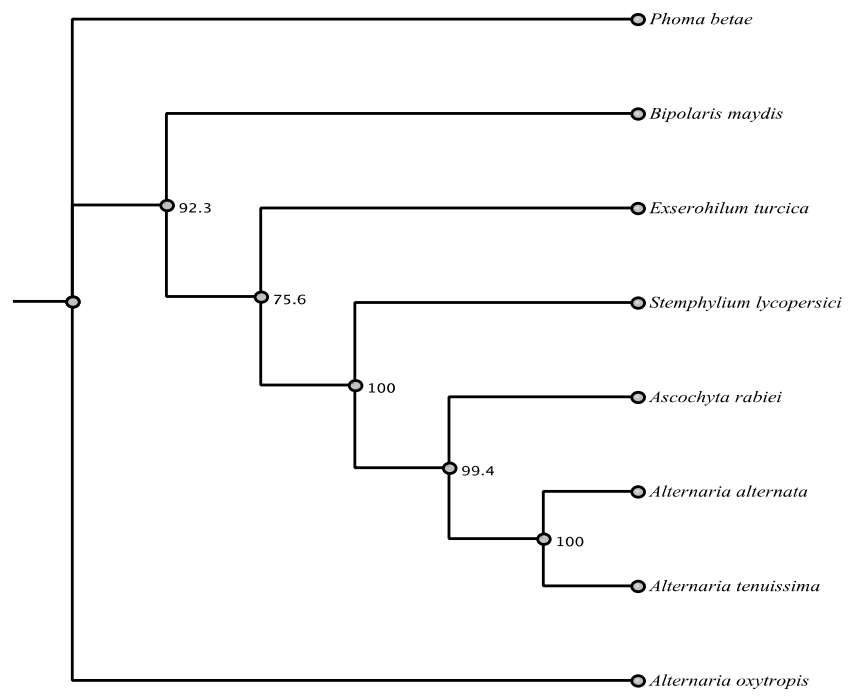

Figure 4. Maximum parsimony tree (MUSCLE alignment and 1000 replicates). Bipolaris maydis was used as the outgroup. Fungi in tree are pblast results of 96133 sequence.

The highly reducing PKS 39849 was most similar to phenolpthiocerol polyketide synthase $p p s A$ of Pyrenophora teres (Pleosporaceae, Pleosporales) with $71.3 \%$ nucleotide 
identity and $74.5 \%$ amino acid identity (Figure S10). The closest BLASTp matches are within the Pleosporaceae and all fall within the Pleosporales (Table 6, Figure 5).

Table 6. Percent amino acid (aa) identity of pblast results of PKS 39849 sequence. Fungal order within the class Dothideomycetes.

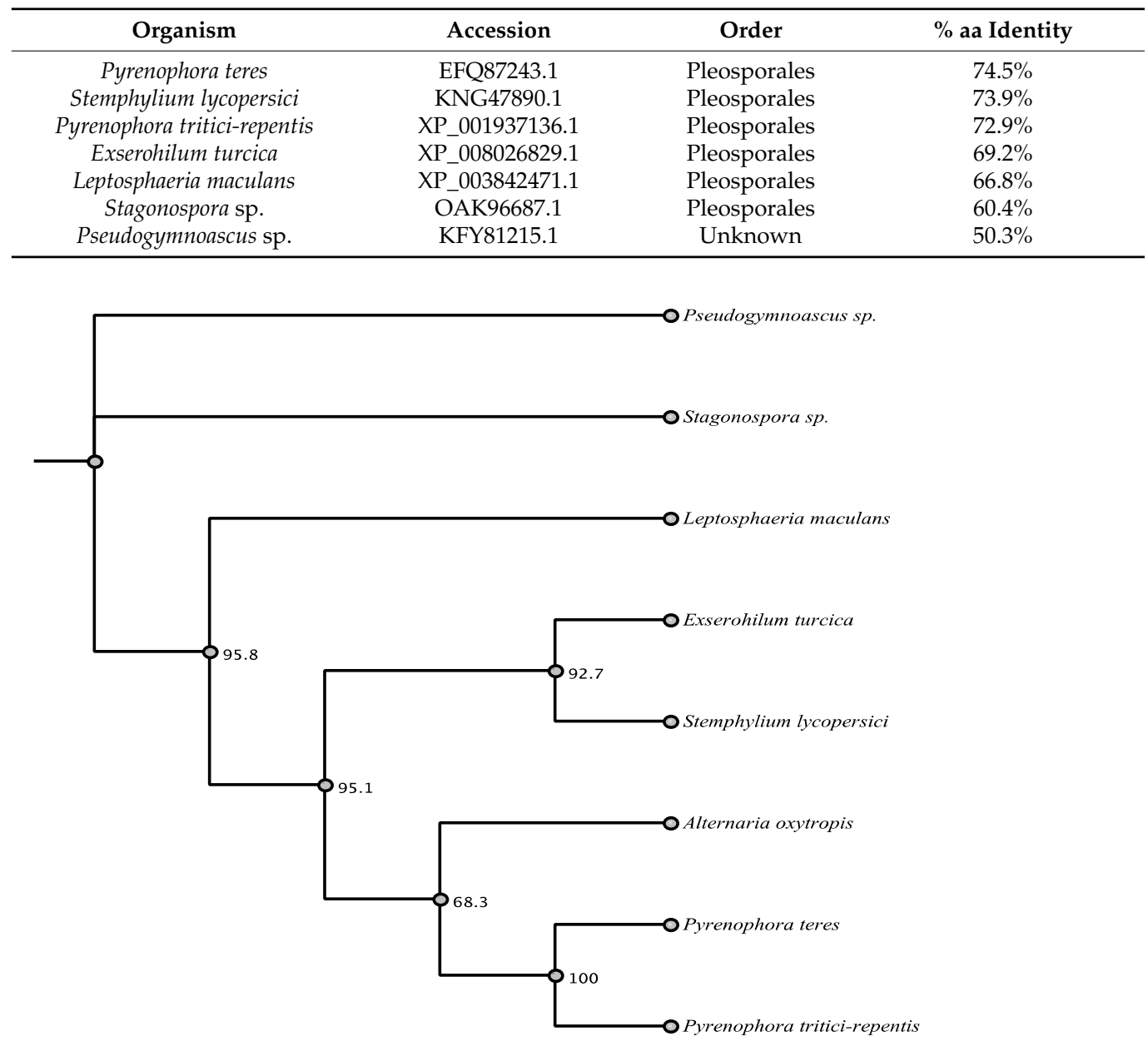

Figure 5. Maximum parsimony tree (MUSCLE alignment and 1000 replicates). Stagonospora sp. was used as the outgroup. Fungi in tree are pblast results of 39849 sequence.

\subsubsection{Partially Reducing Type I PKS}

The partially reducing PKS 17612 has 64\% amino acid identity with a hypothetical protein from Penicillium antarcticum (Eurotiomycetes) and 63\% identity with Cladosporium cladosporioides (Dothideomycetes) cla2 PPSB. CLA2 encodes the highly reducing polyketide synthase cladosporin biosynthesis cluster. None of the top seven BLASTp matches fell within the order Pleosporales and only one fell within the Dothideomycetes (Table S2, Figures S11 and S23).

PKS 8407 has $80.2 \%$ nucleotide identity with the $p k s F$ polyketide synthase of Alternaria solani (Pleosporaceae, Pleosporales) by BLASTn. Amino acid identity with PKSF, a highly reducing PKS, was $81 \%$. PKS 8407 contains the domains KS, AT, DH, ER, ACP and CDD match shows domains that are very similar (Figure S12). PKS 8407 had moderately conserved amino acid identity of $69.4-81.1 \%$ with the top seven matches that were all Alternaria spp. or relatives (Table S3, Figure 6).

PKS 3398 was most closely related by BLASTn to a partial mRNA from Aspergillus eucalypticola (Eurotiomycetes) (66\% identity), Epichloe festucae Fl1 (Sordariomycetes) (66\% identity), Metarhizium robertsii beta-ketoacyl synthase (Sordariomycetes) (66\% identity), and Metarhizium brunneum beta-ketoacyl synthase (68\% identity, but more gaps). The highest 
protein identity was to M. brunneum with $64 \%$ amino acid identity (Figure S14). None of the matches fell within the order Pleosporales, and only one fell within the Dothideomycetes (Figure 7, Table S4).

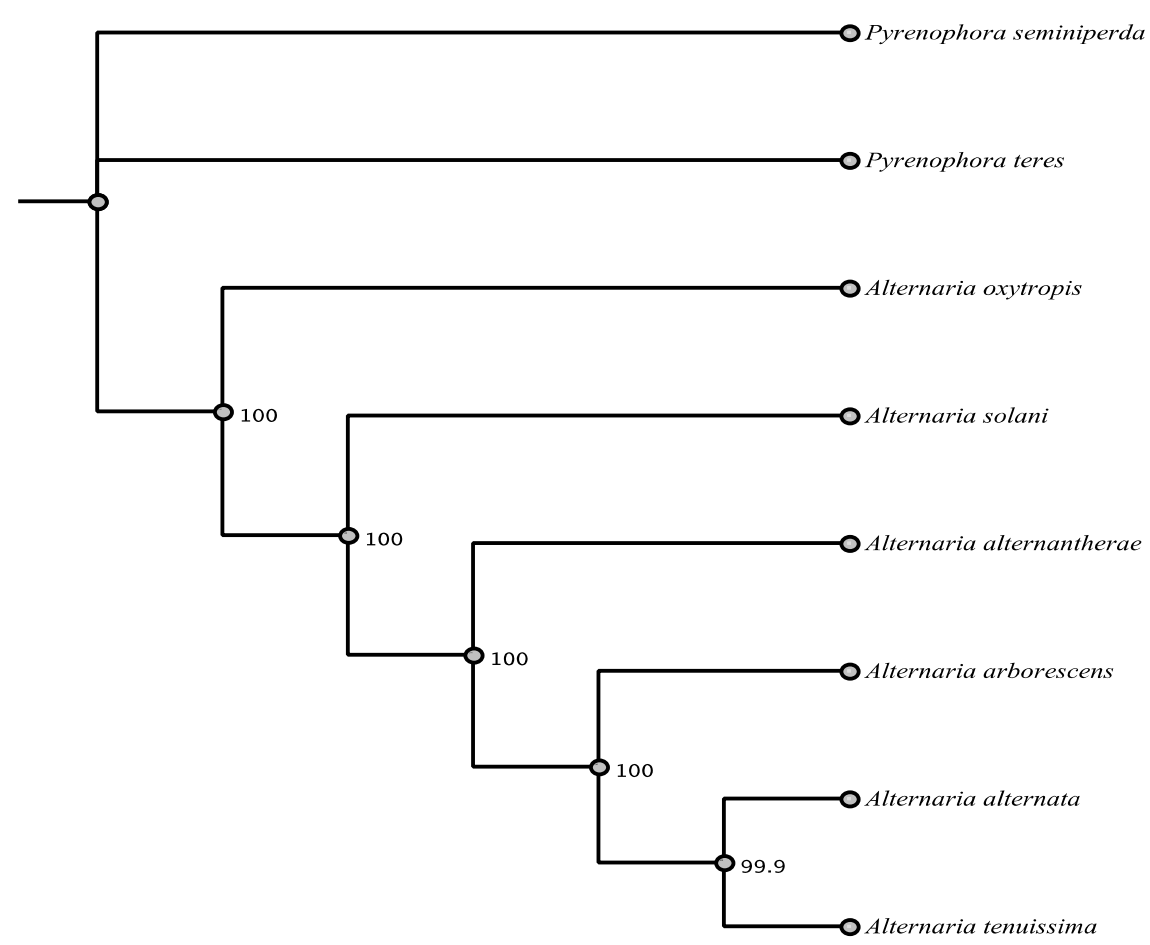

Figure 6. Maximum parsimony tree (MUSCLE alignment and 1000 replicates). Pyrenophora seminiperda was used as the outgroup. Fungi in tree are pblast results of 8407 sequence.

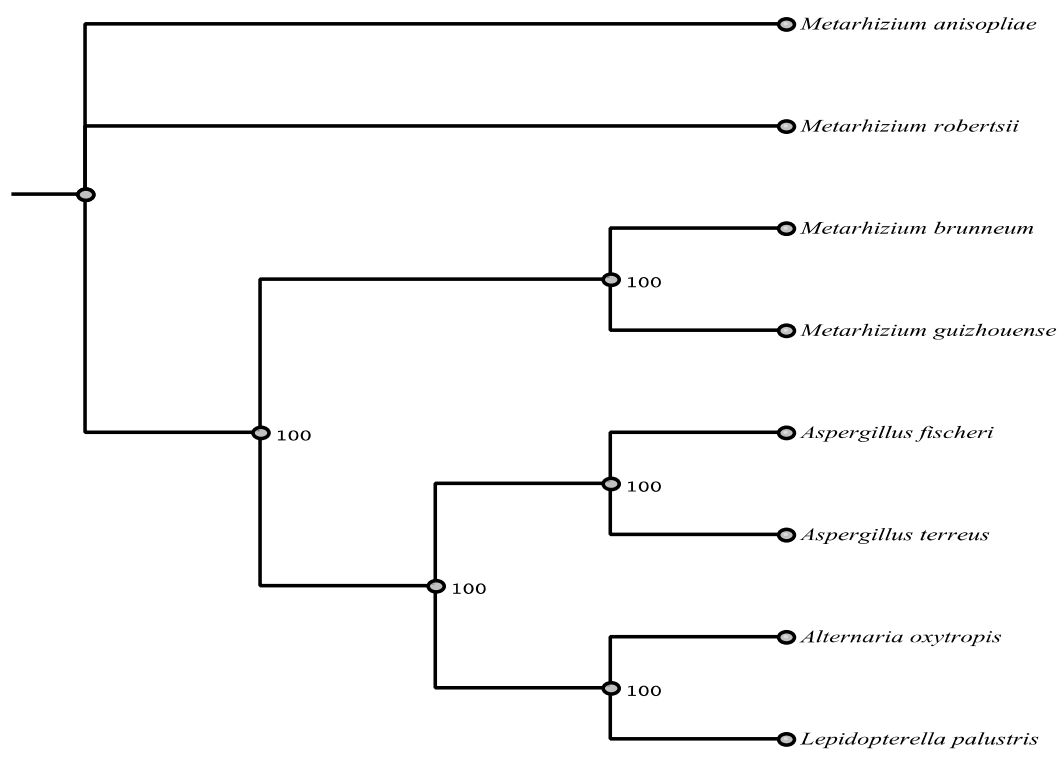

Figure 7. Maximum parsimony tree (MUSCLE alignment and 1000 replicates). Aspergillus fischeri was used as the outgroup. Fungi in tree are pblast results of 3398 sequence.

\subsubsection{Non-Reducing Type I PKS}

The non-reducing Type I PKS 40283 has high nucleotide (86.6\%) and amino acid (95\%) identity to pksA of Alternaria alternata (Pleosporaceae, Pleosporales). PKS 40283 has the domains KS, AT, two ACP, and TE and the domain order is identical by CDD (Figure S13). Prediction of secondary metabolite products indicated that PKS 40283 likely 
produced melanin (Table 2), thus PKS 40283 is likely responsible for melanin production in A. oxytropis. The identity match with PKSA of Alternaria alternata, along with other melanin producing PKS from fungi, leads to this function for PKS 40283. PksA in A. alternata functions as the biosynthetic pathway for melanin production [31,32]. PKS 40283 was very highly conserved had very high amino acid identity of $88.9-95.3 \%$ and the top five matches were Alternaria spp. (Table S5, Figure 8).

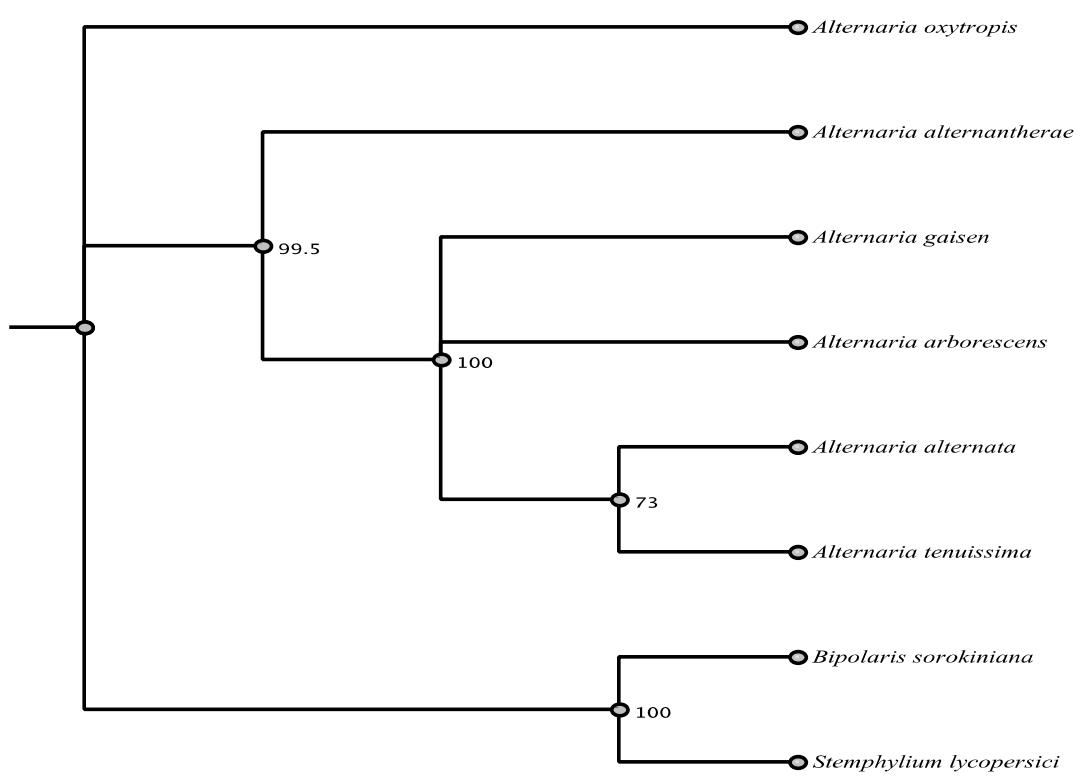

Figure 8. Maximum parsimony tree (MUSCLE alignment and 1000 replicates). Stemphylium lycoperScheme 40283 sequence.

PKS 29103 was most closely related by BLASTn to a gene encoding a hypothetical protein of Thielavia terrestris (Sordariomycetes) with 65.9\% identity (Figure S22). A BLASTp search yielded a hypothetical protein from Epicoccum nigrum (Pleosporaceae, Pleosporales) with $70 \%$ amino acid identity and $p k s 27$, which codes for Asparasone A from Aspergillus flavus (Eurotiomycetes) with 61\% identity.

\subsection{Non-Ribosomal Peptide Synthases}

Of the five non-ribosomal peptide synthases found in the A. oxytropis genome, the function of NRPS 33635 is best understood because of its $84.6 \%$ nucleotide identity with the NPS6 gene of Alternaria alternata (Pleosporaceae, Pleosporales) and 91\% amino acid identity with Alternaria alternata NRPS by BLASTp. NRPS 33635 has the domains A, PCP, $\mathrm{C}$, two PCPs, and $\mathrm{C}$ with both CDD and antiSMASH. Comparison with CDD shows that the proteins are almost identical (Figure S15). NRPS 33635 was highly conserved and had high amino acid identity of $82.6-91.5 \%$ and the top 4 matches that were Alternaria spp. and the next three matches were Pleosporales (Table S6, Figure 9). Contig 33635 (Table 2, Figure S15), was predicted to cyclize. It has an adenylation domain (A), peptidyl carrier protein (PCP, or PP-in the figures), and ends with a condensation-like domain (plain condensation domain in figures). This agrees with the predicted product of dimethyl coprogen, a siderophore produced by A. longipes [33].

NRPS 5682 had 85\% identity with Alternaria brassicae nrps1 gene by BLASTn. BLASTp gave the highest matches with the NRPS1 of $A$. alternata with $91 \%$ amino acid identity and A. brassicae with $89 \%$ identity. NRPS 5682 represents a partial sequence and so contains fewer domains than are found for A. brassicae NRPS1 (Figure S16). Domains in the following order: A, PCP, C, A, PCP, E, C, and A were identified for NRPS 5682. NRPS5682 was highly conserved and had high amino acid identity of 79.2-90.8\% with the top seven matches, which were all Alternaria spp. or relatives (Table S7, Figure S24). NRPS 8194 showed the 
highest nucleotide match from BLASTn and BLASTp searches to an mRNA of Pyrenophora tritici repentis (Pleosporaceae, Pleosporales) HC-toxin synthetase with $85 \%$ nucleotide identity and $87 \%$ amino acid identity. NRPS 8194 has the domains PCP, C, A, PCP, C, and A with both CDD and antiSMASH. Comparison on CDD of HC-toxin synthetase with NRPS8194 shows that the domains are similar but NRPS 8194 could be a truncated form of HC-toxin synthetase (Figure S18). All protein matches fell within the Pleosporaceae. Contigs 5682 (Figure S16) and 8194 (Figure S18), are partial contigs that are closely related to AAP78735 and XP_001939433, respectively. These close matches have an A domain, C-domain, PP domain, and end with a C-like domain. NRPS 5682 is closest match to HC toxin synthetase, a cyclic tetrapeptide, which agrees with the predicted cyclization. In contrast, little is known about the structure of the nrps1 gene of A. brassicae, which is the closest match to NRPS 8194.

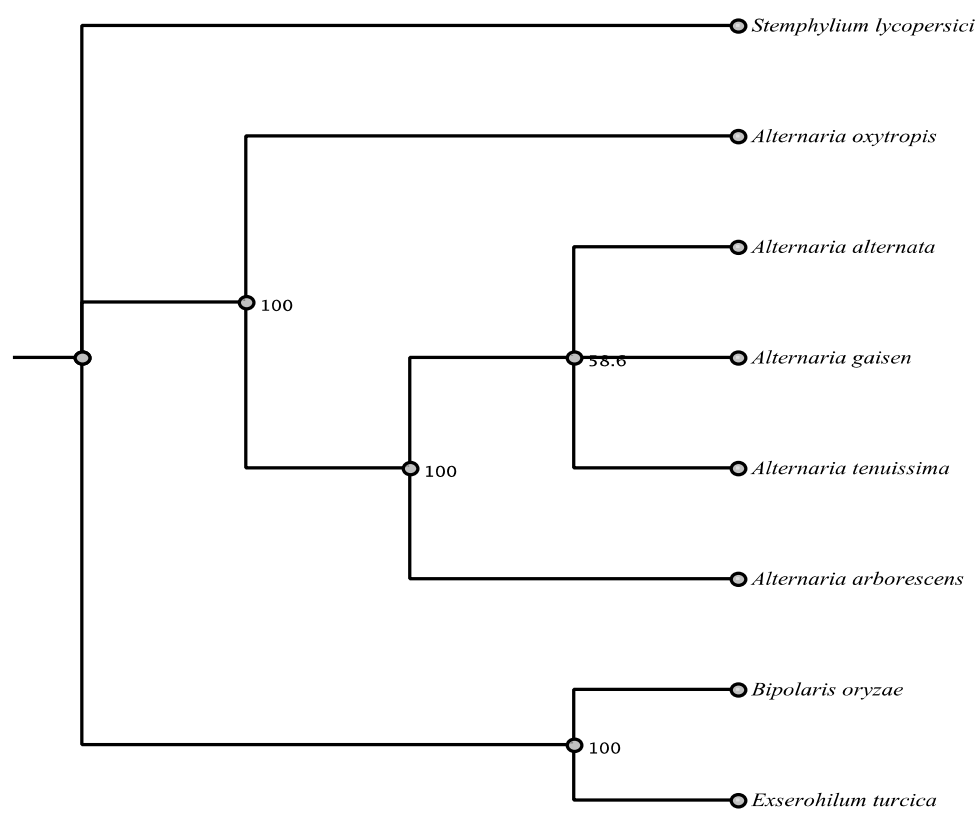

Figure 9. Maximum parsimony tree (MUSCLE alignment and 1000 replicates). Bipolaris oryzae was used as the outgroup. Fungi in tree are pblast results of 33635 sequence.

NRPS 7859 had $82.7 \%$ nucleotide identity and $76 \%$ amino acid identity with the nrps9 gene from Bipolaris maydis (Pleosporaceae, Pleosporales). NRPS 7859 contains the domains $\mathrm{A}, \mathrm{PCP}, \mathrm{C}$, and A with both CDD and antiSMASH and comparison with CDD also showed similarity of domain order (Figure S17). The top four protein matches fell within the Pleosporaceae and the rest within the Eurotiomycetes. NRPS 40703 showed highest BLASTn and BLASTp matches with NRPS2 of Alternaria alternata (Pleosporaceae, Pleosporales) with $82 \%$ nucleotide identity and $82 \%$ amino acid identity. Contig 40703 (Figure S19), lacks a PP (PCP) domain, so is not predicted to cyclize and the final product of NRPS 40703 has not been identified.

\subsection{A Domain Substrates}

The adenylation domain is a catalytic domain that is important for the biosynthesis of natural product peptides. The substrate engages first with the A domain before it is incorporated with the peptide natural product [34]. Every A domain selects a unique substrate $[24,26]$. Prediction of the A domain substrates (Table 2) showed that almost every A domain had at least one Phe. Two contigs were exceptions; for contigs 58,882 and 62407 , the predicted substrates were Gly and Tyr, respectively. Some contigs had multiple A domains, each with its predicted substrate. Contig 5682 was predicted to contain three A domains, each with a Phe substrate, contig 7859 also had three A domains with substrates Pro, Try, Try (in that order), and contig 40703 had four A domains with substrates 
aminoadipate, Phe, Phe, Val, (in that order). Contig 33635 (Table 2), had Phe as a predicted substrate which is consistent with the predicted product of dimethyl coprogen. Contig 8194 was predicted to contain three A domains with substrates Try, Pro, Phe, however the closest match, HC toxin synthase has 4 domains and one uses Pro as a substrate and two use Ala. Since a final SM product has not been determined for NRPS 5682, 7859 and 40703, prediction of their A substrates cannot be verified.

\subsection{Phylogenetic Relationships}

Analysis of the closest relationships for each SM tested revealed surprising levels of diversity. Alternaria falls within the family Pleosporaceae, the order Pleosporales, and the class Dothideomycetes; $68 \%$ of the secondary metabolites gave a closest top match with the same genus, family, order, or class as A. oxytropis. Phylogenetic relationships among closest amino acid matches with Alternaria sp. were for seven highly conserved (81-95\% aa identity) PKS and NRPS: PKS 2122, PKS 96133, PKS 8407, PKS 40283, NRPS 33635, NRPS 5682, and NRPS 40703. These PKS and NRPS, marked as " $A$ " in Table 1, are all well characterized with known functions and clear inheritance lines. Five SM were most closely related to other members within the Pleosporaceae family; PKS-NRPS 58882, PKS 1562, PKS 39849, NRPS 7859, and NRPS 8194. These were marked as "P" in Table 1 and showed intermediate to high conservation (76-87\% aa identity) with the top match. Three PKS and PKS-NRPS, PKS-NRPS 5601, PKS-NRPS 21438, and PKS 29103, were most closely related to a single member of the Pleosporales order with the remaining matches to fungi that did not fall within the Dothideomycetes. These were marked as " $\mathrm{M}$ " in Table 1 and showed intermediate to high conservation (69-88\% aa identity) with the top match. Seven PKS and PKS-NRPS showed no identity with any members of the Pleosporales or the class Dothideomycetes; PKS-NRPS 62407, PKS 59499, PKS 9132 (Table S8, Figures S9 and S25), PKS 12778 (Figure S20), PKS 3398, and PKS 42460 (Figure S21) showed low to intermediate conservation (64-73\% aa identity) with their closest match.

\section{Discussion}

The number of PKSs identified in this work for A. oxytropis, 22, is lower compared to some toxin-producing fungi. Aspergillus species have been identified with high numbers of PKS and NRPS gene clusters (33-81) [8,10,16] and Fusarium species with 26-32 gene clusters [15]. Lu et al. [35] found motifs for 59 possible PKSs for A. oxytropis, but did not further confirm or identify them, and thus the higher number could be due to pseudogenes.

The presence of KSD and CD (Table 2) correlated with the presence of PKS, and condensation domains as shown in the figures. The contigs (Supplemental Figures and Table 2, show the presence of a KS domain. Contigs from Figures S2-S4 and S15-S20, and Table 2 contain a condensation domain. Both $C$ and KS domains are highly conserved. Type I PKS, modular and hybrid, are described by C and KS domain phylogeny [25].

Cyclization of final peptide product requires the presence of a condensation (C), adenylation (A), and end with a thiolation (T) domains. Fungi NRPS lack a terminal thioester (TE) domain. Instead, macrocyclic fungal NRPS end with a condensation-like domain (Ct), which corresponds, in function, to TE [36]. Requirements for cyclization include the presence of a C domain, A domain, T domain (PCP, peptidyl carrier protein), and a terminal $\mathrm{Ct}$ domain (with or without an attached PP arm) [36-39]. Cyclization predictions were confirmed for the NRPS in which a product had been identified. NRPS 33635 satisfied all requirements for cyclization and the product is cyclized. NRPS 8194 is a partial sequence, and its closest matches fulfills the cyclization requirements in producing a cyclic tetrapeptide. Similarly, NRPS 5682 is a partial sequence, and its closest match fulfills the cyclization requirements, however the identity of the product has not been confirmed. Though some other contigs included all three domains required for cyclization, they did not end with a Ct domain, or lacked a PCP domain. NRPS 40703 (Figure S19), lacks a PP (PCP) domain, but has an ACP-like domain. While ACP domains (acyl-carrier protein) 
resemble the T domain of NRPS [40] it is not required for cyclization, which suggests that this contig would not enzymatically cyclize its end product.

The A domain of NRPS are required for the biosynthesis of the peptide natural product, and substrate selection is determined by the A domain. All basic NRPS must have an A domain, required for the biosynthesis of the peptide natural product, and substrate selection; a PCP (thiolation) domain, transports and attaches the substrates to different catalytic domains; and a $\mathrm{C}$ domain, that catalyzes peptide bond formation $[34,40]$. While amino acids substrates were predicted, since few final products have been identified, it was difficult to determine the accuracy of some of the predictions.

Seven of the 22 PKSs identified were most similar to an Alternaria species. This was not unexpected as A. oxytropis is most closely related to other Alternaria species and Alternaria oxytropis falls within the Pleosporaceae. Among the seven PKSs most similar to other Alternaria species, they were distributed among the highly reducing PKS, partially reducing PKS, nonreducing PKS, and NRPS. Other fungi with closest matches within the Pleosporaceae were Pyrenophora, Bipolaris, and Setsosphaeria. There were four PKS from the genus Pyrenophora. Bipolaris spp. provided the closest match for two PKS. Fusarium with two matches was the fungus outside of the Pleosporaceae with the highest number of matches.

Several of the PKS/NRPS identified (PKS 2122, PKS 8407, NRPS 5682) showed high identity with Alternaria species or relatives through the first seven matches and three others, PKS 40283, PKS 96133, and NRPS 33635) showed high identity with Alternaria species and other Pleosporales. These PKS and NRPS are obviously highly conserved among Alternaria sp. and Pleosporales. For several of the sequences, including PKS 8407, NRPS 3365, A. oxytropis clustered with other Alternaria spp. in the phylogenetic trees. In other phylogenetic trees (PKS 40283, PKS 96133) A. oxytropis did not cluster with other Alternaria spp.

For A. oxytropis, $68 \%$ of the SM genes did not match the genus Alternaria even at the amino acid level. Four sequences (PKS-NRPS 58882, PKS-NRPS 21438, NRPS 8194, and PKS 39849) had moderately high identity with Pyrenophora species in the family Pleosporaceae, and the rest of the matched fungi did not fall within the order Pleosporales. Two of those (PKS-NRPS 21438 and NRPS 8194) showed high conservation with 87-88\% aa identity with Pyrenophora spp. Most Pyrenophora are pathogenic on cereals and the genus is monophyletic [41]. Pyrenophora is a sexual state of what was previously known as Drechslera. Even for sequences in which there is high identity with Alternaria species, such as PKS 2122 and NRPS 5682, the phylogenetic trees suggest that $A$. oxytropis is as closely related to Pyrenophora as to Alternaria spp. This raises questions about the inheritance of these SM and the taxonomic placement of the fungus within the genus Alternaria. This high sequence conservation suggests a potential taxonomic lineage for Alternaria oxytropis, perhaps as a product of hybridization or recombination between Alternaria and Pyrenophora ancestors.

Three sequences analyzed, PKS 9132, PKS 17612, and PKS 3398, had no closest matches in the Pleosporales. For the PKS 9132 sequence, the phylogenetic trees show no clustering of $A$. oxytropis with any of the closest matches, whereas for the other two sequences, $A$. oxytropis clustered with otherwise unrelated fungi.

Within the phylum Ascomycota, the large majority of SM gene clusters are found only in closely related species [8]. Those few that are more broadly distributed across the phylum are often highly divergent between even closely related species [22]. Rokas et al. [42] suggested several possible explanations for SM cluster variability based on molecular evolutionary processes including functional diversity, horizontal gene transfer, and de novo assembly. For A. oxytropis SM, functional diversity is likely, horizontal gene transfer is possible, and no evidence was found for de novo assembly.

Orthologous or paralagous functional diversity could explain some of the variability found in A. oxytropis SM, particularly for those with the closest matches to members of the Pleosporaceae, but not Alternaria, marked as P in Table 1. All five of these SM genes showed slight divergence in domain size or order from their closest matches. NRPS 8194 
differs slightly in gene order from that of the HC-toxin synthetase gene of Pyrenophora tritici-repentis and A. oxytropis is not known to produce HC toxin. The SWN gene cluster of swainsonine-producing fungi vary in the order of genes other than swnK (PKS-NRPS 58882) in the cluster [6].

The A. oxytropis SM genes that did not match fungi within the order Pleosporales or did not match fungi within the class Dotheomycetes have a more speculative ontogeny. Some of the variability could be to due to horizontal gene transfer or more likely a combination of horizontal gene transfer and orthologous or paralagous changes. The domains (and order of domains) in these A. oxytropis SM genes are similar to their closest matching fungi. Horizontal gene transfer between fungal classes has been speculated for the entire sterigmatocystin biosynthetic pathway, that appears to have been transferred from Aspergillus nidulans to Podospora [43]. A study of SM within Aspergillus fumigatus showed that 13 of the SM gene clusters were generally conserved with low variation and 23 were highly variable [8]. Those authors found six examples of gene content polymorphisms that were exemplified by loss of gene cluster function, structural changes in the metabolite, or change in the expression or transport of the metabolite.

Manning et al. [44] reported that the genome of Pyrenophora tritici-repentis includes several NRPSs that may have been derived by horizontal gene transfer and gene duplication. The polyketide host-selective toxins associated with Alternaria spp. adapted to pear, apple, tangerine, citrus, rough lemon, and tomato are all found on conditionally dispensable chromosomes (CDC) that could have been acquired through horizontal chromosome transfer among the Alternaria spp. [45]. Armitage et al. [46] showed that while A. tenuissima pathotypes shared 10 types of transposable elements (TE) with $A$. arborescens, the pathogens contained significantly decreased numbers of TE in the DDE and gypsy families, and significantly higher numbers of TE in the mariner family. They speculated that the TEs may have increased the variability in the fungi. Determining if gain of whole gene clusters through TE or horizontal chromosome transfer might be possible explanations for SM sequences in A. oxytropis would require many more sequences from related species, chromosome mapping of the fungus, analysis of the number and location of TE, and functional analyses of the SM.

The closest matches for the A. oxytropis PKSs were for Alternaria species for highly conserved genes. PKS40283 had the highest amino acid identity (95\%) of any of the PKSs identified, with A. alternata PKSA, which is responsible for melanin production. The gene responsible for melanin production is highly conserved among many dark-colored fungi. Although A. oxytropis is dark black in culture and A. cinerea and A. fulva are tan and grey, respectively, in culture [29], the latter two fungi have the same sequence as identified for PKS40283. Silencing of the homologous gene, $p k s 1$, in Slafractonia leguminicola, also within the family Plesoporaceae and a swainsonine toxin producer, caused a reduction in melanin synthesis and relevant transcript levels [47].

NRPS 3365 also had high amino acid homology (91\%) with Alternaria alternata NPS6. This is likely a highly conserved NRPS among both pathogenic and saprobic fungi, including pathogenic and nonpathogenic isolates of Pyrenophora tritici-repentis [44]. NPS6 of Cochliobolus spp. was found to have orthologs in all filamentous fungi examined [48]. It was also found to affect virulence since loss of expression reduced virulence but did not completely abolish it. Additionally, lack of expression led to an increase in sensitivity to hydrogen peroxide. In Alternaria alternata, nps6 is necessary for the biosynthesis of dimethyl copreogen siderophores as well as functioning as a virulence factor [49]. This was the predicted SM for A. oxytropis for NRPS 3365 as well. Multiple transcription factors control nps6 transcript accumulation in A. alternata including NADPH oxidase, a redox responsive transcription facto YAP1 and a mitogen activated protein kinase HOG1 [50]. Mutation of $n p s 6$ or any of the transcription factors results in increased sensitivity to reactive oxygen species (ROS) and reduced virulence in citrus. ROS sensitivity can be partially rescued though with the addition of iron. The effects of iron and ROS sensitivity lead to the idea that NPS6 is important to the production of siderophores involved in iron uptake [50]. 
Deletion of NPS6 in Fusarium graminearum, Cochliobolus miyabeanus, and A. brassicicola also resulted in the same responses of increased sensitivity to ROS and reduced virulence [51].

NRPS 5682 also showed the same high level of amino acid identity (91\%) with $A l$ ternaria alternata NRPS1. Alternaria NRPS1 is likely involved in plant infection since increased expression of nrps1 was found during host infection by A. brassicae [52]. This NRPS could play some role in the establishment of the endophytic relationship of $A$. oxytropis in its plant host. PKS2122 showed a high level of amino acid identity (90\%) with Alternaria solani PKSN. PKSN is the product of the alt5 gene, which is essential for alterapyrone biosynthesis [30]; when alt5 was expressed in Aspergillus oryzae under an alpha-amylase promoter, alternapyrone was produced. Because there was a high level of identity at both the nucleotide and protein levels between PKS2122 and PKSN, it is likely that A. oxytropis also produces this compound. PKS2122 was very highly conserved among many Alternaria species. PKS-NRPS 5882 was verified as the swnK KS [6]. Two of the fungi with high amino acid identity, Pyrenophora seminiperda and Clohesyomyces aquaticus have not been reported to produce swainsonine or be associated with mammalian toxicity.

Both the number of PKS, along with the lack of associated functions identified here, are common to studies in other fungi. While additional PKS genes or PKS-like genes might be identified from the completed genome sequence of $A$. oxytropis, it is likely that the majority of the PKS genes have been identified here. Interestingly, the functions of the 22 genes found in this study remain unknown. Although secondary metabolites have been studied in many fungi, and the genomes of many fungi have been sequenced, it is significant that functions for these have not been readily identified. Transcriptomic and metabolomic studies will likely be necessary to better understand the function of the genes identified in this work.

\section{Conclusions}

Secondary metabolite production is essential to most fungi, and especially so to those fungi that produce toxins. The PKS, NRPS, and PKS/NRPS identified for A. oxtryopis show the conservation of these genes within the genus Alternaria and order Pleosporales. The PKS gene for melanin exemplifies very high conservation. The data presented here also highlight the lack of information on how these genes are derived, spread, and diversified. The PKS and PKS/NRPS identified here that have no close matches within the Dothideomycetes are examples that need additional work to help explain their ontogeny. Unless a fungus is economically important, it is unlikely to have been sequenced. Sequencing of many other fungi within the Dothideomycetes will help to identify some conservation between the poorly characterized secondary metabolite genes and more highly characterized PKS, NRPS, and PKS/NRPS, and thus closing the knowledge gap.

Supplementary Materials: The following are available online at https: / www.mdpi.com/article / 10.3390/jof7070538/s1, Figure S1: Domain order of PKS-NRPS 58882 and polyketide synthase of Metarhizium acridum XP_07815889, Figure S2: Domain order of PKS-NRPS 5601 and hypothetical protein of Setosphaeria turcica XP_008025924, Figure S3: Domain order of PKS-NRPS 21438 and hypothetical protein of Pyrenophora teres XP_003303559, Figure S4: Domain order of PKS-NRPS 62407 and hypothetical protein of Tolypocladium ophioglossoides KND87119, Figure S5: Domain order of PKS 2122 and ALT5 of Alternaria solani BAD83684, Figure S6: Domain order of PKS 1562 and PKS5 of Bipolaris maydis AAR90268, Figure S7: Domain order PKS 59499 and hypothetical protein of Aspergillus nomius KNG90368, Figure S8: Domain order of PKS 96133 and PKSF of Alternaria alternata AFN68297, Figure S9: Domain order of PKS 9132 and PKSD of Aspergillus niger XP_001394543, Figure S10: Domain order PKS 39849 and PPSA of Pyrenophora tritici-repentis XP_001937136, Figure S11: Domain order of PKS 17612 and PPSB of Pyrenothora tritici-repentis XP_001934720, Figure S12: Domain order of PKS 8407 and Alternaria solani PKSF BAE80697, Figure S13: Domain order of PKS 40283 and PKSA of Alternaria alternata AFN68292, Figure S14: Domain order of PKS 3398 and polyketide synthase of Metarhizium brunneum KID62944, Figure S15: Domain order of NRPS 33635 and NPS6 of Alternaria alternata AFN69082, Figure S16: Domain order of PKS 5682 and Alternaria brassicae nrps1 AAP78735, Figure S17: Domain order of NRPS 7859 and NPS9 of Cochliobolus heterostrophus AAX09991, Figure S18: 
Domain order of NRPS 8194 and HC-toxin synthetase of Pyrenophora tritici-repentis, Figure S19: Domain order of NRPS 40703 and NPS2 of Alternaria brassicicola, Figure S20: Domain order of PKS 12778 and hypothetical protein from Fusarium oxysporum $f$. sp. conglutinans EXL67078, Figure S21: Domain order of PKS 42460 and hypothetical polyketide synthase of Colletotrichum gloeosporioides XP_007270670, Figure S22: Domain order of PKS 29103 and hypothetical protein of Aspergillus parasiticus KJK63007, Figure S23: Maximum parsimony tree (MUSCLE alignment and 1000 replicates). Lasiodiplodia theobromae was used as the outgroup. Fungi in tree are pblast results of 17612 sequence; Figure S24: Maximum parsimony tree (MUSCLE alignment and 1000 replicates). Pyrenophora teres was used as the outgroup. Fungi in tree are pblast results of 5682 sequence.: Figure S25: Maximum parsimony tree (MUSCLE alignment and 1000 replicates). Aspergillus luchuensis was used as the outgroup. Fungi in tree are pblast results of 9132 sequence. Table S1: GenBank accession numbers and amino acid percent identity of blast results of 2122 sequence, Table S2: Genbank accession numbers and amino acid percent identity of blast results of 17612 sequence, Table S3: Genbank accession numbers and amino acid percent identity of blast results of 8407 sequence, Table S4: Genbank accession numbers and amino acid percent identity of blast results of 3398 sequence, Table S5: GenBank accession numbers and amino acid percent identity of blast results of 40283 sequence, Table S6: Genbank accession numbers and amino acid percent identity of blast results of 33635 sequence, Table S7: Genbank accession numbers and amino acid percent identity of blast results of 5682 sequence, Table S8: Genbank accession numbers and amino acid percent identity of blast results of 9132 sequence.

Author Contributions: Conceptualization, D.B.H., R.C., C.L.S., D.C.; methodology, D.B.H., C.L.S., D.C., R.C.; analysis, D.B.H., M.N., T.N., C.L.S., R.C.; writing, D.B.H., R.C., D.C.; supervision, R.C. All authors have read and agreed to the published version of the manuscript.

Funding: This research received no external funding.

Institutional Review Board Statement: Not applicable.

Informed Consent Statement: Not applicable.

Data Availability Statement: Sequence data has been deposited into Genbank. See Table 1 for specific accession numbers.

Conflicts of Interest: The authors declare no conflict of interest.

\section{References}

1. Stegelmeier, B.L.; Lee, S.T.; James, L.F.; Gardner, D.R.; Panter, K.E.; Ralphs, M.H.; Pfister, J.A. The Comparative Pathology of Locoweed Poisoning in Livestock, Wildlife and Rodents. In Poisonous Plants: Global Research and Solutions; CABI Publishing: Wallingford, UK, 2007; pp. 359-365.

2. James, L.F.; Van Kampen, K.R.; Staker, G.R. Locoweed (Astragalus lentiginosus) poisoning in cattle and horses. J. Am. Veter Med Assoc. 1969, 155, 525-530.

3. Cook, D.; Grum, D.S.; Gardner, D.R.; Welch, K.D.; Pfister, J.A. Influence of endophyte genotype on swainsonine concentrations in Oxytropis sericea. Toxicon 2013, 61, 105-111. [CrossRef] [PubMed]

4. Harris, C.M.; Schneider, M.J.; Ungemach, F.S.; Hill, J.E.; Harris, T.M. Biosynthesis of the toxic indolizidine alkaloids slaframine and swainsonine in Rhizoctonia leguminicola: Metabolism of 1-hydroxyindolizidines. J. Am. Chem. Soc. 1988, 110, 940-949. [CrossRef]

5. Patrick, M.; Adlard, M.W.; Keshavarz, T. Production of an indolizidine alkaloid, swainsonine by the filamentous fungus, Metarhizium anisopliae. Biotechnol. Lett. 1993, 15, 997-1000. [CrossRef]

6. Cook, D.; Donzelli, B.; Creamer, R.; Baucom, D.L.; Gardner, D.R.; Pan, J.; Moore, N.; Krasnoff, S.B.; Jaromczyk, J.W.; Schardl, C.L. Swainsonine Biosynthesis Genes in Diverse Symbiotic and Pathogenic Fungi. Genes Genomes Genet. 2017, 7, 1791-1797. [CrossRef]

7. Noor, A.I.; Neyaz, M.; Cook, D.; Creamer, R. Molecular Characterization of a Fungal Ketide Synthase Gene Among SwainsonineProducing Alternaria Species in the USA. Curr. Microbiol. 2020, 77, 2554-2563. [CrossRef]

8. Lind, A.; Wisecaver, J.H.; Lameiras, C.; Wiemann, P.; Palmer, J.; Keller, N.; Rodrigues, F.; Goldman, G.; Rokas, A. Drivers of genetic diversity in secondary metabolic gene clusters within a fungal species. PLoS Biol. 2017, 15, e2003583. [CrossRef]

9. Hertweck, C. The Biosynthetic Logic of Polyketide Diversity. Angew. Chem. Int. Ed. 2009, 48, 4688-4716. [CrossRef]

10. Ahuja, M.; Chiang, Y.-M.; Chang, S.-L.; Praseuth, M.B.; Entwistle, R.; Sanchez, J.F.; Lo, H.-C.; Yeh, H.-H.; Oakley, B.R.; Wang, C.C.C. Illuminating the Diversity of Aromatic Polyketide Synthases in Aspergillus nidulans. J. Am. Chem. Soc. 2012, 134, 8212-8221. [CrossRef]

11. Maiya, S.; Grundmann, A.; Li, X.; Li, S.-M.; Turner, G. Identification of a Hybrid PKS/NRPS Required for Pseurotin a Biosynthesis in the Human PathogenAspergillus fumigatus. ChemBioChem 2007, 8, 1736-1743. [CrossRef] 
12. Boddy, C.N. Bioinformatics tools for genome mining of polyketide and non-ribosomal peptides. J. Ind. Microbiol. Biotechnol. 2014, 41, 443-450. [CrossRef]

13. Chooi, Y.-H.; Tang, Y. Navigating the Fungal Polyketide Chemical Space: From Genes to Molecules. J. Org. Chem. 2012, 77, 9933-9953. [CrossRef]

14. Boettger, D.; Hertweck, C. Molecular Diversity Sculpted by Fungal PKS-NRPS Hybrids. ChemBioChem 2012, 14, 28-42. [CrossRef]

15. Hansen, F.T.; Sørensen, J.L.; Giese, H.; Søndergaard, T.; Frandsen, R.J. Quick guide to polyketide synthase and nonribosomal synthetase genes in Fusarium. Int. J. Food Microbiol. 2012, 155, 128-136. [CrossRef]

16. Inglis, O.D.; Binkley, J.; Skrzypek, M.S.; Arnaud, M.B.; Cerqueira, G.C.; Shah, P.; Wymore, F.; Wortman, J.R.; Sherlock, G. Comprehensive annotation of secondary metabolite biosynthetic genes and gene clusters of Aspergillus nidulans, A. fumigatus, A. niger and A. oryzae. BMC Microbiol. 2013, 13, 91. [CrossRef]

17. Liu, L. Bioactive metabolites from the plant endophyte Pestalotiopsis fici. Mycology 2011, 2, 37-45. [CrossRef]

18. Wang, X.; Zhang, X.; Liu, L.; Xiang, M.; Wang, W.; Sun, X.; Che, Y.; Guo, L.; Liu, G.; Guo, L.; et al. Genomic and transcriptomic analysis of the endophytic fungus Pestalotiopsis fici reveals its lifestyle and high potential for synthesis of natural products. BMC Genom. 2015, 16, 1-13. [CrossRef]

19. Kearse, M.; Moir, R.; Wilson, A.; Stones-Havas, S.; Cheung, M.; Sturrock, S.; Buxton, S.; Cooper, A.; Markowitz, S.; Duran, C.; et al. Geneious Basic: An integrated and extendable desktop software platform for the organization and analysis of sequence data. Bioinformatics 2012, 28, 1647-1649. [CrossRef]

20. Conway, K.R.; Boddy, C.N. ClusterMine360: A database of microbial PKS/NRPS biosynthesis. Nucleic Acids Res. 2012, 41, D402-D407. [CrossRef]

21. Geer, L.Y.; Marchler-Bauer, A.; Geer, R.C.; Han, L.; He, J.; He, S.; Liu, C.; Shi, W.; Bryant, S.H. The NCBI BioSystems database. Nucleic Acids Res. 2009, 38, D492-D496. [CrossRef] [PubMed]

22. Khaldi, N.; Seifuddin, F.T.; Turner, G.; Haft, D.; Nierman, W.C.; Wolfe, K.H.; Fedorova, N.D. SMURF: Genomic mapping of fungal secondary metabolite clusters. Fungal Genet. Biol. 2010, 47, 736-741. [CrossRef]

23. Blin, K.; Shaw, S.; Steinke, K.; Villebro, R.; Ziemert, N.; Lee, S.Y.; Medema, M.H.; Weber, T. antiSMASH 5.0: Updates to the secondary metabolite genome mining pipeline. Nucleic Acids Res. 2019, 47, W81-W87. [CrossRef]

24. Medema, M.H.; Blin, K.; Cimermancic, P.; De Jager, V.; Zakrzewski, P.; Fischbach, M.A.; Weber, T.; Takano, E.; Breitling, R. antiSMASH: Rapid identification, annotation and analysis of secondary metabolite biosynthesis gene clusters in bacterial and fungal genome sequences. Nucleic Acids Res. 2011, 39, W339-W346. [CrossRef] [PubMed]

25. Ziemert, N.; Podell, S.; Penn, K.; Badger, J.H.; Allen, E.; Jensen, P.R. The Natural Product Domain Seeker NaPDoS: A Phylogeny Based Bioinformatic Tool to Classify Secondary Metabolite Gene Diversity. PLoS ONE 2012, 7, e34064. [CrossRef]

26. Prieto, C.; Garcia-Estrada, C.; Lorenzana, D.; Martín, J.F. NRPSsp: Non-ribosomal peptide synthase substrate predictor. Bioinformatics 2012, 28, 426-427. [CrossRef]

27. Jones, P.; Binns, D.; Chang, H.-Y.; Fraser, M.; Li, W.; McAnulla, C.; McWilliam, H.; Maslen, J.; Mitchell, A.; Nuka, G.; et al. InterProScan 5: Genome-scale protein function classification. Bioinformatics 2014, 30, 1236-1240. [CrossRef] [PubMed]

28. Zierep, P.F.; Padilla, N.; Yonchev, D.G.; Telukunta, K.K.; Klementz, D.; Günther, S. SeMPI: A genome-based secondary metabolite prediction and identification web server. Nucleic Acids Res. 2017, 45, W64-W71. [CrossRef]

29. Baucom, D.L.; Romero, M.; Belfon, R.; Creamer, R. Two new species of Undifilum, fungal endophytes of Astragalus (locoweeds) in the United States. Botany 2012, 90, 866-875. [CrossRef]

30. Fujii, I.; Yoshida, N.; Shimomaki, S.; Oikawa, H.; Ebizuka, Y. An Iterative Type I Polyketide Synthase PKSN Catalyzes Synthesis of the Decaketide Alternapyrone with Regio-Specific Octa-Methylation. Chem. Biol. 2005, 12, 1301-1309. [CrossRef]

31. Fetzner, R.; Seither, K.; Wenderoth, M.; Herr, A.; Fischer, R. Alternaria alternata transcription factor CmrA controls melanization and spore development. Microbiology 2014, 160, 1845-1854. [CrossRef]

32. Kimura, N.; Tsuge, T. Gene cluster involved in melanin biosynthesis of the filamentous fungus Alternaria alternata. J. Bacteriol. 1993, 175, 4427-4435. [CrossRef] [PubMed]

33. Jalal, M.A.F.; Love, S.K.; van der Helm, D. Na-Dimethylcoprogens, Three novel trihydoxamate siderophones from pathogenic fungi. Biol. Met. 1988, 1, 4-8. [CrossRef] [PubMed]

34. Miller, B.R.; Gulick, A.W. Structural biology of non-ribosomal peptide synthetases. Methods Mol. Biol. 2016, 1401, 3-29. [CrossRef] [PubMed]

35. Lu, H.; Quan, H.; Ren, Z.; Wang, S.; Xue, R.; Zhao, B. The Genome of Undifilum oxytropis Provides Insights into Swainsonine Biosynthesis and Locoism. Sci. Rep. 2016, 6, 30760. [CrossRef]

36. Gao, X.; Haynes, S.W.; Ames, B.D.; Wang, P.; Vien, L.P.; Walsh, C.T.; Tang, Y. Cyclization of fungal nonribosomal peptides by a terminal condensation-like domain. Nat. Chem. Biol. 2012, 8, 823-830. [CrossRef] [PubMed]

37. Bills, G.; Li, Y.; Chen, L.; Yue, Q.; Niu, X.-M.; An, Z. New insights into the echinocandins and other fungal non-ribosomal peptides and peptaibiotics. Nat. Prod. Rep. 2014, 31, 1348-1375. [CrossRef]

38. Bloudoff, K.; Schmeing, T.M. Structural and functional aspects of the nonribosomal peptide synthetase condensation domain superfamily: Discovery, dissection and diversity. Biochim. Biophys. Acta Proteins Proteom. 2017, 1865, 1587-1604. [CrossRef]

39. Yun, C.-S.; Motoyama, T.; Osada, H. Biosynthesis of the mycotoxin tenuazonic acid by a fungal NRPS-PKS hybrid enzyme. Nat. Commun. 2015, 6, 8758. [CrossRef] 
40. Khayatt, B.I.; Overmars, L.; Siezen, R.J.; Francke, C. Classification of the Adenylation and Acyl-Transferase Activity of NRPS and PKS Systems Using Ensembles of Substrate Specific Hidden Markov Models. PLoS ONE 2013, 8, e62136. [CrossRef]

41. Ariyawansa, H.; Kang, J.; Chukeatirote, E.; Hyde, K. Pyrenophora. Mycosphere 2014, 5, 351-362. [CrossRef]

42. Rokas, A.; Mead, M.E.; Steenwyk, J.L.; Raja, H.A.; Oberlies, N.H. Biosynthetic gene clusters and the evolution of fungal chemodiversity. Nat. Prod. Rep. 2020, 37, 868-878. [CrossRef]

43. Slot, J.C.; Rokas, A. Horizontal Transfer of a Large and Highly Toxic Secondary Metabolic Gene Cluster between Fungi. Curr. Biol. 2011, 21, 134-139. [CrossRef]

44. Manning, V.A.; Pandelova, I.; Dhillon, B.; Wilhelm, L.J.; Goodwin, S.; Berlin, A.M.; Figueroa, M.; Freitag, M.; Hane, J.K.; Henrissat, B.; et al. Comparative Genomics of a Plant-Pathogenic Fungus, Pyrenophora tritici-repentis, Reveals Transduplication and the Impact of Repeat Elements on Pathogenicity and Population Divergence. Genes Genomes Genet. 2013, 3, 41-63. [CrossRef]

45. Akagi, Y.; Akamatsu, H.; Otani, H.; Kodama, M. Horizontal Chromosome Transfer, a Mechanism for the Evolution and Differentiation of a Plant-Pathogenic Fungus. Eukaryot. Cell 2009, 8, 1732-1738. [CrossRef]

46. Armitage, A.; Cockerton, H.M.; Sreenivasaprasad, S.; Woodhall, J.; Lane, C.R.; Harrison, R.; Clarkson, J.P. Genomics Evolutionary History and Diagnostics of the Alternaria alternata Species Group Including Apple and Asian Pear Pathotypes. Front. Microbiol. 2020, 10, 3124. [CrossRef]

47. Alhawatema, M.S.; Gebril, S.; Cook, D.; Creamer, R. RNAi-mediated down-regulation of a melanin polyketide synthase (pks1) gene in the fungus Slafractonia leguminicola. World J. Microbiol. Biotechnol. 2017, 33, 179. [CrossRef]

48. Lee, B.-N.; Kroken, S.; Chou, D.Y.T.; Robbertse, B.; Yoder, O.C.; Turgeon, B.G. Functional Analysis of All Nonribosomal Peptide Synthetases in Cochliobolus heterostrophus Reveals a Factor, NPS6, Involved in Virulence and Resistance to Oxidative Stress. Eukaryot. Cell 2005, 4, 545-555. [CrossRef]

49. Chen, L.-H.; Lin, C.-H.; Chung, K.-R. A nonribosomal peptide synthetase mediates siderophore production and virulence in the citrus fungal pathogenAlternaria alternata. Mol. Plant Pathol. 2013, 14, 497-505. [CrossRef]

50. Chen, L.-H.; Yang, S.L.; Chung, K.-R. Resistance to oxidative stress via regulating siderophore-mediated iron acquisition by the citrus fungal pathogen Alternaria alternata. Microbiology 2014, 160, 970-979. [CrossRef]

51. Oide, S.; Moeder, W.; Krasnoff, S.; Gibson, D.; Haas, H.; Yoshioka, K.; Turgeon, B.G. NPS6, Encoding a Nonribosomal Peptide Synthetase Involved in Siderophore-Mediated Iron Metabolism, Is a Conserved Virulence Determinant of Plant Pathogenic Ascomycetes. Plant Cell 2006, 18, 2836-2853. [CrossRef]

52. Guillemette, T.; Sellam, A.; Simoneau, P. Analysis of a nonribosomal peptide synthetase gene from Alternaria brassicae and flanking genomic sequences. Curr. Genet. 2004, 45, 214-224. [CrossRef] [PubMed] 